\title{
Evaluación rápida de la avifauna en el Centro de Investigación, Posgrado y Conservación Amazónica (CIPCA), provincia de Napo, Amazonía Ecuatoriana
}

\section{Rapid evaluation of the birdlife at the Research, Postgraduate and Amazon Conservation Center (CIPCA), Napo province, Ecuadorian Amazon}

\author{
Wilmer A. Shiguango-Yumbo ${ }^{1}$, Carolina Bañol-Pérez ${ }^{2}$ \\ ${ }^{1}$ Universidad Estatal Amazónica. Puyo, Pastaza, Ecuador \\ ${ }^{2}$ Facultad de Ciencias de la Vida, Universidad Estatal Amazónica, Pastaza, Ecuador \\ wilmershiguango@yahoo.com,cbanol@uea.edu.ec
}

\begin{abstract}
Resumen
$\mathrm{E}^{1}$ Centro de Investigación Posgrado y Conservación Amazónica de la Universidad Estatal Amazónica se encuentra ubicado en la vertiente oriental ecuatoriana de la Cordillera de los Andes, cubriendo un área aproximada de 2840 ha de bosques primario y secundario. La presente investigación se basó en hacer un inventario rápido de aves en estratos de bosque secundario y en estratos de zonas intervenidas (silvopastoriles y agropecuarias) del Cipca. Se realizaron conteos por transectos mediante observación y grabaciones de cantos para registrar las especies, los cuales se realizaron de forma intensiva durante los meses de abril y mayo del 2018. Se registraron un total de 94 especies en ambas zonas de estudio correspondientes a 30 familias, siendo las más abundantes Thraupidae (27.6\%) y Tyrannidae (16\%) aves muy comunes que habitan las zonas intertropicales. Los estratos de bosque secundario fueron los más diversos con un índice de Margalef de 10.5 y un índice de Shannon de 3.6. Sin embargo, la zona intervenida nos muestra que existe mayor abundancia referente al número de individuos por especie, quizás debido a la presencia de especies arbóreas frutales en el lugar. También se determinaron las especies en categorías de riesgo según la Unión Internacional para la Conservación de la Naturaleza, resultando 13 especies con grado de alto valor para estas categorías, las cuales deben considerarse importantes para programas de conservación.
\end{abstract}

Palabras claves: aves, monitoreo, diversidad, vulnerabilidad, conservación

Rec.: 14.05.2019. Acept.: 27.01.2020. Publicado el 30 de junio de 2020

\begin{abstract}
The Research, Postgraduate and Amazon 1 Conservation Center of the Amazon State University is located on the eastern side of the Ecuadorian Andes Mountains, covering an area of approximately 2840 hectares of primary and secondary forests. The aim of the present investigation was an inventory rapid of birds in strata of secondary forest and in strata of intervened areas (silvopastoral and agricultural interventions). Transect counts were made by observation and call recordings to register the species, which were done intensively during the months of April and May 2018. A total of 94 species were recorded in both study areas corresponding to 30 families, being the most abundant Thraupidae (27.6\%) and Tyrannidae (16\%) which are very common birds in intertropical zones. Secondary forest strata were the most diverse with a Margalef index of 10.5 and a Shannon index of 3.6. However, the intervened area shows a greater abundance regarding the number of individuals per species, perhaps due to the presence of fruit tree species. Species in risk categories were also determined according to International Union for Conservation of Nature, resulting in 13 species with high value for these categories, which should be considered important for conservation programs.
\end{abstract}

Keywords: birds, monitoring, diversity, vulnerability, conservation 


\section{Introducción}

$\mathrm{E}$ cuador es uno de los países con mayor biodiversidad de aves en el mundo, el cual alberga 1690 especies de aves, después de Colombia y Perú (Mindo Cloud Fundation, 2017). Las aves son parte integral de los ecosistemas de bosques amazónicos (Daily, 1997), son el grupo más conocido en su aspecto ecológico, taxonómico y por su valor como indicadores de la calidad ambiental; pero desafortunadamente, la diversidad ecuatoriana y los ecosistemas están siendo alterados por diversos factores antrópicos (Freile, 2019). En las regiones amazónicas, la ganadería, los monocultivos, la extracción y deforestación de especies maderables, el tráfico ilegal de vida silvestre está causando la fragmentación del componente biótico de los ecosistemas. Estas actividades pueden ser consideradas una amenaza para las aves a escala regional, porque ocasiona la perdida de sitios de reproducción y de espacios de concentración de las comunidades que llegan en las épocas migratorias (Naranjo, 1998). La avifauna presenta diferentes grados de sensibilidad frente a las alteraciones del hábitat (alta, media y baja sensibilidad). Las especies de baja sensibilidad son aquellas que pueden adaptarse con facilidad a ambientes alterados; especies de mediana sensibilidad las que pueden encontrarse en zonas alteradas y no alteradas, y por último las de alta sensibilidad, aves que habitan bosques en buen estado de conservación (Stotz et al., 1996).

En la última década se ha incrementado el número de investigaciones ornitológicas en la región amazónica, gracias a iniciativas individuales, institucionales o al surgimiento de grupos de observación de aves. Sin embargo, la información obtenida es poca para entender la dinámica ecológica de estas comunidades (Freile, 2019).

El Cipca alberga bosque siempre verde montano del Norte de la Cordillera de los Andes (MAE, 2012), lo que proporciona un sitio interesante para mantener sus ecosistemas conservados y permitir realizar estudios de diversidad de especies y de servicios ecosistémicos. La falta de investigaciones de avifauna en el sitio de estudio se evidencia en la poca o inexistente información acerca de la riqueza o diversidad de estas comunidades. Por lo tanto, las evaluaciones rápidas son una herramienta importante para establecer una línea base de la composición de especies que permitan diagnosticar áreas de importancia, así como especies de interés para la conservación en sitios donde no existen estudios o son deficientes (Sayre et al., 2002; Losada et al., 2005; RAMSAR 2005; Molina et al., 2015).

Consecuentemente, se plantea realizar un inventario rápido de especies de aves, con la finalidad de determinar la abundancia y diversidad en dos tipos de bosques presentes (bosque secundario poco intervenido y bosque con programas silvopastoriles y agropecuarios), lo que permitirá conocer especies claves para determinar el estado de los ecosistemas y de las especies sensibles a la fragmentación de bosques.

\section{Materiales y Métodos}

\section{Zona de estudio}

La investigación fue realizada en el Centro de Investigación Posgrado y Conservación Amazónica Cipca, localizado en el Cantón Arosemena Tola de la Provincia de Napo, Amazonía Ecuatoriana, localizado en el kilómetro 44, vía Puyo-Tena entre los ríos Anzu y Piatúa (Figura 1). El Cipca tiene un total de 2848.20 hectáreas de superficie, de las cuales 2093.90 corresponden a bosque primario, 445.91 hectáreas corresponden a bosque secundario y 274.43 corresponden a la zona de programas didácticos y agropecuarios (UEA, 2018). La zona de estudio se caracteriza por tener zonas ligeramente onduladas sin pendientes pronunciadas, registra pluviosidades de $4000 \mathrm{~mm}$ anuales aproximadamente, una temperatura promedio de 15 a $25^{\circ} \mathrm{C}$ y una humedad relativa del 80\%; esta zona es un área de transición entre la amazonia alta y la amazonia baja con altitudes que varían entre los 580 a $990 \mathrm{msnm}$.

\section{Monitoreo de especies}

Los registros de especies se realizaron en lugares de bosque secundario y en partes de la zona intervenida ( 3 transectos cada uno) por un periodo de 40 días entre los meses de abril y mayo del 2018. Se utilizaron dos métodos para el levantamiento de la información: conteo por transectos y grabaciones.

Los transectos se realizaron en las dos áreas de estudio: bosque secundario y zona Intervenido. Cada transecto presentó una longitud de $1 \mathrm{~km}$ y fue establecido en senderos existentes para disminuir el impacto en la vegetación. Se realizó el recorrido durante las horas de mayor actividad de las aves (de $07 \mathrm{~h} 00$ am a $11 \mathrm{~h} 00 \mathrm{am}$ y de $14 \mathrm{~h} 00$ a $16 \mathrm{~h} 00 \mathrm{pm}$ ), con un tiempo de recorrido de una hora por kilómetro (Villareal, et al., 2004). Se identificaron aves mediante la detección auditiva y la observación visual directa mediante binoculares Bushnell (10x42), la guía de Aves del Ecuador (Ridgely y Greenfield, 2010) y la guía Fieldbook of the Birds of Ecuador including the Galápagos Islands (Mc Mullan y Navarrete, 2010).

Las grabaciones se realizaron usando una grabadora digital Voice Recorder VN-100PC, en cada uno de los tres transectos, se grabaron las vocalizaciones de las especies que tuvieron dificultad para la identificación en las dos áreas de estudio. Para la identificación de las vocalizaciones se utilizó el banco de sonidos del Ecuador (Moore et al., 2003). 


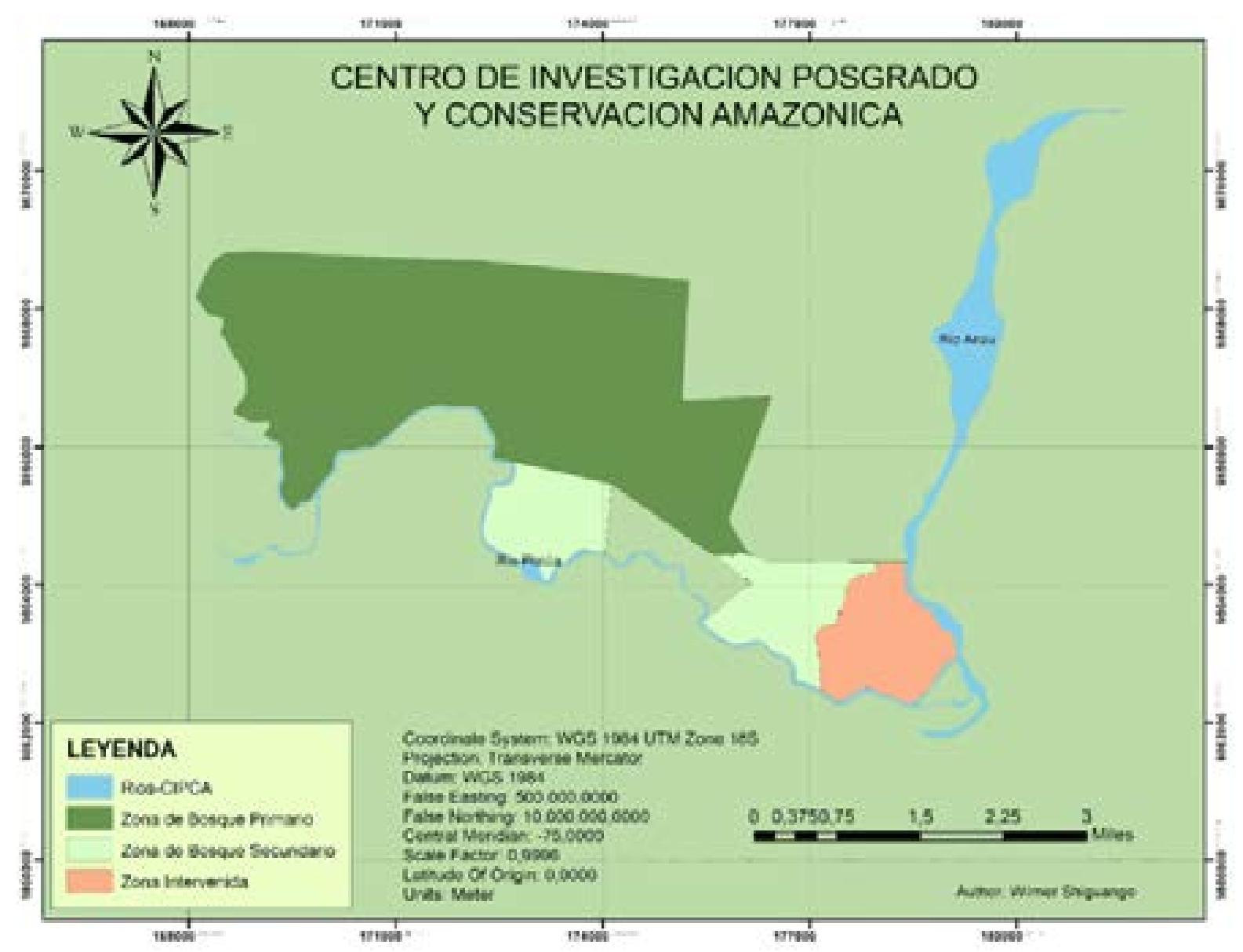

Figura 1. Mapa del área de estudio del Centro de Investigación, Posgrado y Conservación Amazónica. Los puntos representan las zonas de monitoreo

\section{Determinación taxonómica}

Para determinar las especies registradas se siguió la propuesta del South American Classification Committe, American Ornitologists Unión (Remsen et al., 2017) y para nombres en español, se usaron los propuestos por el Comité Ecuatoriano de Registros Ornitológicos (Freile et al., 2017).

También se compilaron bases de datos con registros disponibles de las aves registradas en Pastaza y Napo, mediante fuentes bibliográficas, recursos electrónicos como e-Bird, Eco registros, CERO, listados de nombres comunes y registros del grupo científico de monitoreo de aves de la amazonia ecuatoriana, entre otras.

\section{Análisis de datos}

\section{Abundancia}

La abundancia relativa se calculó como el número de individuos observados de cada especie, divididos para el número de individuos observados de todas las especies en cada transecto de cada estrato analizado. Este resultado multiplicado por cien corresponde al porcentaje que contribuye cada especie a la comunidad. La riqueza de especies de cada estrato se calculó como el número total de especies detectadas (todas las especies observadas y escuchadas en los tres transectos de cada estrato) (Niemi y McDonald, 2004).

\section{Curva de acumulación de especies}

Las curvas de acumulación de especies se utilizan para estimar el número de especies a partir de un muestreo a modo de determinar la representatividad del mismo. Representa la manera en el cual las especies van apareciendo en las unidades de muestreo, o de acuerdo con el incremento en el número de individuos. Para realizar las curvas de acumulación de especies se utilizó el programa EstimateS 9.1.0 (Colwell, 2013), el cual toma los datos provenientes de un sistema de muestreo estandarizado, aleatoriza toda la información y realiza cálculos del número de especies observadas y esperadas utilizando estimadores y considerando las desviaciones estándar provenientes del proceso de aleatorización (Villareal et al., 2004). Se utilizaron estimadores de riqueza no paramétricos, debido a que no asumen el tipo 
de distribución del conjunto de datos y no los ajusta a un modelo determinado. Específicamente se utilizaron los estimadores: Chao 1 y ACE. El estimador Chao 1 se refiere a la abundancia de individuos que pertenecen a una determinada clase de muestra. El estimador se basa en cuantas especies estas representadas de un solo individuo en la muestra (sigletons), y cuantas especies están representadas por dos individuos (doubletons):

$$
\mathrm{S}_{\text {est }}=\mathrm{S}_{\mathrm{obs}}+\mathrm{F}^{2} / 2 \mathrm{G}
$$

Dónde,

$\mathrm{S}_{\text {est: }}$ Es el número de especies

$\mathrm{S}_{\text {obs: }}$ Es el número de especies observado en una muestra,

F: Es el número de sigletons

G: Es el número de doubletons

El estimador ACE basado en la abundancia de las especies, que se refiere a la suma de las probabilidades de encontrar especies observadas dentro del total de especies presentes, pero no observadas (Colwell, 2013). El estimador ACE utiliza para las estimaciones para diez o menos individuos por muestra.

\section{Diversidad de especies}

Para analizar la diversidad de la avifauna registrada se utilizaron índices de diversidad, los cuales ayudan a resumir información de las especies en un solo valor. La diversidad local o alfa está basada en la riqueza específica y en la estructura que incorporan valores de abundancia y equitatividad. Los índices calculados fueron:

\section{Índice de Margalef (DMg)}

$$
\mathrm{DMg}=(\mathrm{S}-1) / \mathrm{InN}
$$

Dónde,

$\mathbf{S}$ es el número de especies y $\mathbf{N}$ es el número total de individuos.

\section{Índice de Simpson (1-D)}

$$
\mathrm{D}=\sum \mathrm{pi}^{2}
$$

Dónde,

Pi es la abundancia proporcional de la especie; representa la probabilidad de que un individuo de la especie i esté presente en la muestra, y su recíproco 1-D.

\section{Índice de Shannon Wiener (H)}

$$
\mathrm{H}^{\prime}=-\sum \mathrm{Pi} \ln \mathrm{pi}
$$

Donde,

H' expresa la uniformidad de los valores de importancia, a través de todas las especies de la muestra.

Estos índices de diversidad fueron calculados mediante el programa PAST versión 3.20 (2018) (Øyvind et al., 2001).

\section{Categorías de riesgo}

Para la determinación de las especies registradas en alguna categoría de riesgo, se revisaron las referencias de la Unión Internacional para la Conservación de la Naturaleza (UICN, 2016), el reporte de la Convención sobre el Comercio Internacional de Especies Amenazadas de Fauna y Flora Silvestres de aves del Ecuador (CITES, 2018) y mediante el listado de la Convención Mundial de Aves Migratorias (CMS, 2018).

\section{Resultados y discusión}

\section{Composición de comunidades de aves}

Se registró un total de 737 individuos pertenecientes a 94 especies de aves, que corresponden a 30 familias en toda la zona de estudio, siendo las más abundantes Thraupidae $(27.6 \%)$ y Tyrannidae (16\%), aves muy comunes que habitan las zonas intertropicales (Figura 2). Los dos estratos analizados constituyen remanentes de bosque siempre verde piemontano, donde el $25.1 \%$ de la composición de aves (número de individuos por especie) representó el bosque secundario y el $74.9 \%$ correspondió a la zona intervenida del Cipca.

\section{Abundancia y riqueza de especies}

En lo que corresponde a abundancia se registró en la zona intervenida el mayor número de especímenes logrando obtener un total de 552 individuos y para el bosque secundario un total de 185 individuos, en contraste con el mayor registro de aves que se obtuvo en un bosque tropical caducifolio y el menor registro obtenido en áreas abiertas (Ramirez, 2009).

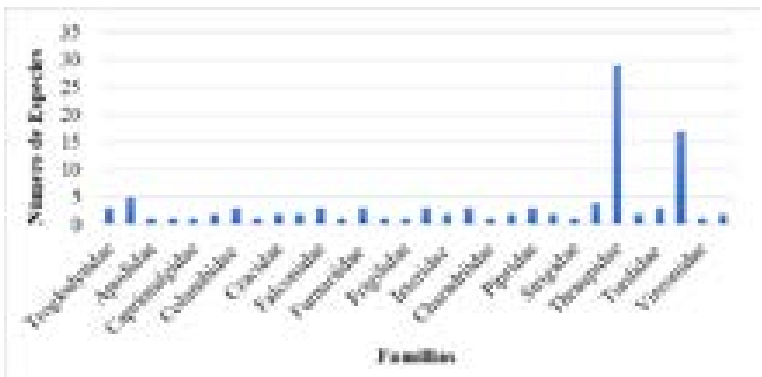

Figura 2. Número de especies de aves por familia registradas en las dos zonas de estudio del Cipca 
Con respecto a la riqueza se logró registrar 53 especies para la zona intervenida y 56 especies para el bosque secundario, destacándose una baja variabilidad de especies en las dos áreas de estudio, lo que indica que la similitud entre hábitats hace que haya un alto intercambio de especies, generando una competencia alimentaria entre las especies presentes y enmarcando su distribución. También la presencia de aves en fragmentos de bosque y cobertura arbórea en los paisajes agrícolas son de gran importancia para la conservación de las aves, porque ellas prefieren la heterogeneidad de usos de la tierra para sus hábitats (Enriquez y Saenz, 2006).

\section{Bosque secundario}

El bosque secundario tiene una extensión de 445.91 hectáreas de bosque, donde se realizaron los monitoreos en tres transectos preestablecidos (senderos), encontrando un total de 185 individuos de 56 especies correspondientes a 23 familias, del cuales las más representativas fueron Thraupidae, Pipridae, Tyrannidae y Accipitridae (Figura 3).

Las especies más representativas de acuerdo a la abundancia relativa dentro de este ecosistema fueron: Tangara chilensis (12.4\%), Streptoprocne zonaris (7.6\%) у Penelope jacquacu (4.9\%) (Cuadro 1).

\section{Bosque intervenido}

El área de bosque intervenida cuenta con una extensión de 274.43 hectáreas, en la cual se realizó el monitoreo en los senderos existentes del área de investigación silvopastoril y agropecuaria, logrando obtener un total de 552 aves de 53 especies correspondientes a 24 familias, de las cuales Thraupidae y Tyrannidae fueron las más abundantes (Figura 4.). Las especies con mayor abundancia relativa fueron Ramphocelus carbo (13.1\%), Psarocolius angustifrons (10.3\%), Crotophaga ani $(9.1 \%)$ y Pionus menstruus (4.2\%) (Cuadro 1).

\section{Curvas de acumulación de especies}

Las curvas de acumulación de especies estimadas para los tres estratos analizados alcanzaron un máximo de 27 especies para el bosque secundario y de 37 especies para la zona intervenida (Figura 5). Lo anterior indica que el esfuerzo de muestreo utilizado registra el $86.52 \%$ para el estimador Chao 1 y $86.69 \%$ para el estimador ACE. Esto nos indica que el esfuerzo de muestreo fue bastante confiable.

La curva de acumulación de especies para el bosque secundario, nos muestra un menor esfuerzo de muestreo representado en $76.83 \%$ para Chao 1 y de $75.23 \%$ para ACE (Figura 6).

Mientras que, para la zona intervenida, los valores fueron más altos, para Chao 1 fue del $99.43 \%$ y para el estimador ACE fue del $97.88 \%$ (Figura 7), lo que indica que en la zona intervenida fue mucho mayor el esfuerzo de muestreo debido a la mayor abundancia de individuos y de especies.

De acuerdo con estos resultados, la riqueza y abundancia de aves es mayor en la zona intervenida con pastos y bovinos, la cual presenta plantas asociadas principalmente árboles frutales, que aumentan el número de hábitats y los períodos de alimentación de diversas especies de aves. Similares resultados se obtuvieron en la provincia del Guayas, donde detallan que la variedad de especies frutales en áreas de intervención antropogénica atraen una gran variedad especies de aves (López, 2015). También otro estudio donde se analizaron tres estratos boscosos, área con pastizal ganadero, bosque en regeneración, y bosque primario se

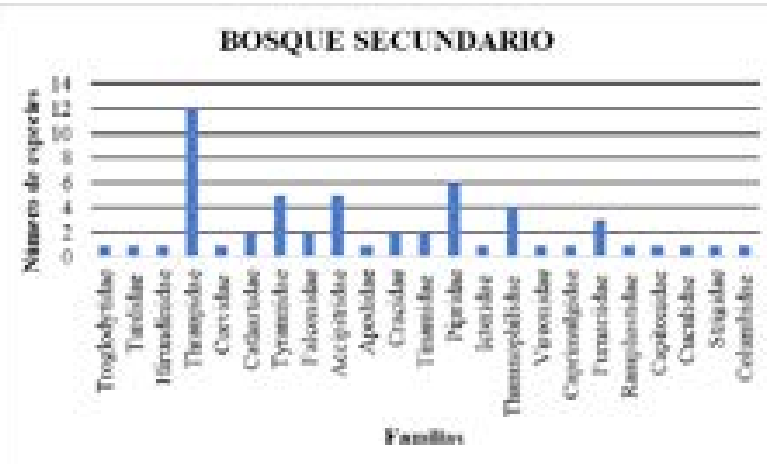

Figura 3. Composición de la avifauna por familias del bosque Secundario

Cuadro 1. Abundancia relativa (AR) de las especies más representativas por cada estrato estudiado. (DE) Desviación estándar.

\begin{tabular}{llcc}
\hline ESTRATO & \multicolumn{1}{c}{ ESPECIES } & AR & DE \\
\hline $\begin{array}{l}\text { Bosque } \\
\text { Secundario }\end{array}$ & Tangara chilensis & 12.43 & 6.65 \\
& $\begin{array}{l}\text { Streptoprocne } \\
\text { zonaris }\end{array}$ & 7.56 & 1.41 \\
& Penelope jacquacu & 4.86 & 4.95 \\
& Ramphocelus carbo & 4.32 & 1.73 \\
& Orlatis guttata & 3.24 & 1.41 \\
Zona & Ramphocelus carbo & 13.04 & 4.60 \\
& $\begin{array}{l}\text { Psarocolius } \\
\text { antervenififrons }\end{array}$ & 10.33 & 8.04 \\
& Crotophaga ani & 9.06 & 21.2 \\
& Pionus menstruus & 4.17 & 0.89 \\
& Coragyps atratus & 3.80 & 4.57 \\
\hline
\end{tabular}




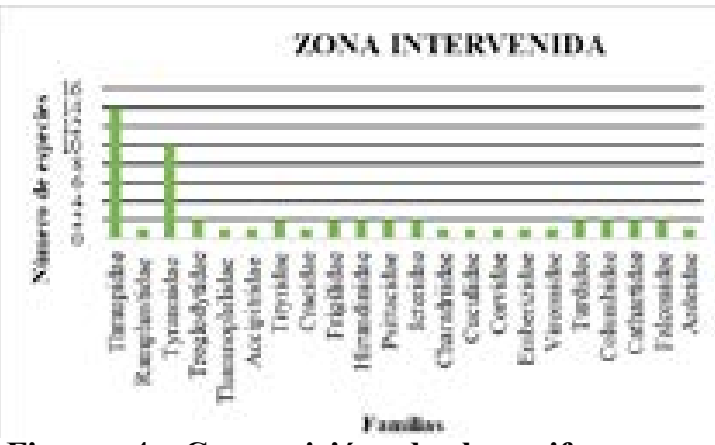

Figura 4. Composición de la avifauna por familias de la zona intervenida

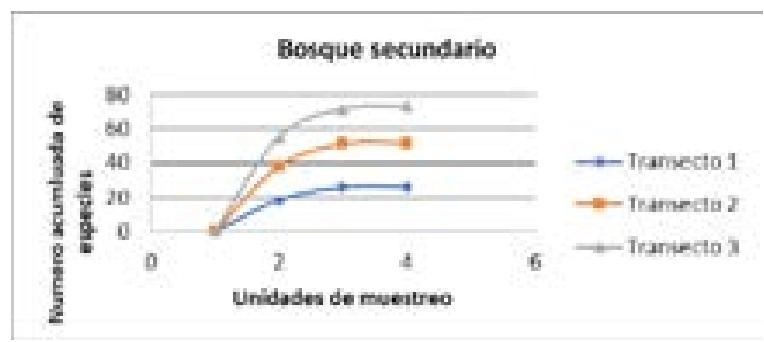

Figura 6. Curva acumulada de especies para el bosque secundario

concluye que la mayoría de aves prefieren el bosque en regeneración por la facilidad de obtener los alimentos (Borojes y Lopez, 2005).

\section{Índices de diversidad}

Se determinaron los respectivos índices de diversidad basados en la riqueza (índice de Margalef) y estructura de las comunidades (índice de Simpson e índice de Shannon). Los resultados basados en el índice de Margalef fueron altos en ambas zonas de estudio lo que representa una alta riqueza de especies para los dos estratos, sin embargo, el bosque secundario presento un valor mayor de 10.5 y la zona intervenida un valor de 7.8 (Cuadro 2).

En este sentido la abundancia y la riqueza de especies no coincidieron, lo que demuestra que más especies prefieren ecosistemas mejor conservados, pero más cantidad de individuos encuentran sus requerimientos ecológicos en zonas asociadas a agroecosistemas (presencia de más especies frutales). Con respecto al índice de Simpson la diferencia entre ambos estratos fue muy poca, quizás debido a que la heterogeneidad de las especies encontradas no fue alta con respecto al número de individuos por especie, sin embargo, la similitud en la composición de las comunidades de aves resulta en un alta de diversidad. El índice de Shannon fue más alto para el bosque secundario (3.6) con una pequeña diferencia para la zona intervenida (3.4), lo que sugiere una similitud en la abundancia de aves en el área de estudio (número de individuos por especie).

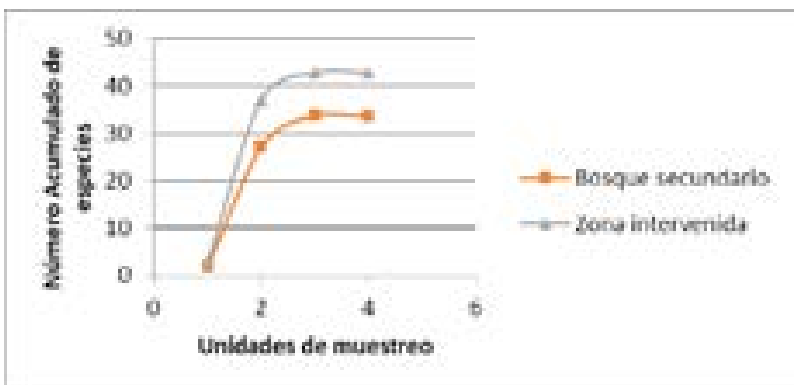

Figura 5. Curva acumulada de especies por estratos analizados en el Cipca

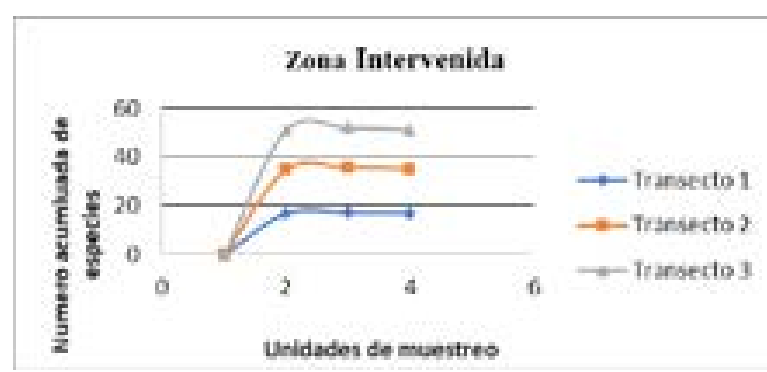

Figura 7. Curva acumulada de especies para la zona intervenida

Cuadro 2. Resultados de índices de diversidad en la comunidad de aves analizada en el Cipca

\begin{tabular}{|c|c|c|}
\hline Índices Ecológicos & $\begin{array}{c}\text { Bosque } \\
\text { Secundario }\end{array}$ & $\begin{array}{c}\text { Zona } \\
\text { Intervenida }\end{array}$ \\
\hline Margalef $(<2,>5)$ & 10.49 & 7.802 \\
\hline $\begin{array}{c}\text { Simpson 1-D (0 } \\
-\overline{1})\end{array}$ & 0.9599 & 0.9474 \\
\hline $\begin{array}{c}\text { Shannon_H (<2. } \\
>\overline{3})\end{array}$ & 3.613 & 3.398 \\
\hline
\end{tabular}

Sin embargo, estos valores altos reflejan que existe un buen número de especies que se correlacionan entre los hábitats, posiblemente debido a la uniformidad de las especies encontradas (Cuadro 2).

\section{Categorías de riesgo}

En cuanto al estado de conservación, se encontraron un total de 13 especies de aves de las cuales 3 especies están incluidas en la lista roja de la UICN en la categoría VU Vulnerable (Tinamus tao, Ramphastos vitellinu y Ramphastos tucanus) y 1 especie en la categoría de NT Casi Amenazado como es el caso de la especie Lepidothrix coronata. También se encontraron 11 especies en el Apéndice II de la Convención sobre el Comercio Internacional de Especies Amenazadas de Fauna y Flora Silvestres de aves y 6 especies bajo el criterio Convención Mundial de Aves Migratorias (Cuadro 3). 


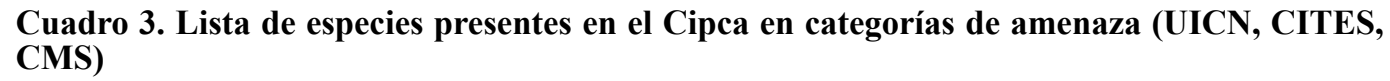

\begin{tabular}{lllll}
\hline$\#$ & \multicolumn{1}{c}{ ESPECIES } & UICN & APENDICE CITES & APENDICE CMS \\
\hline 1 & Tinamus tao & VU & & \\
2 & Black Vulture & LC & II & \\
3 & Turkey Vulture & LC & II & \\
4 & Leptodon cayanensis & LC & II & II \\
5 & Harapagus bidentatus & LC & II & II \\
6 & Rupornis magnirostris & LC & II & II \\
7 & Spizaetus tyrannus & LC & II & II \\
8 & Herpetotheres cachinnans & LC & II & II \\
9 & Falco rufigularis & LC & II & II \\
10 & Vanellus chilensis & LC & & II \\
11 & Aratinga leucophthalmus & LC & II & \\
12 & Pionus menstruus & LC & II & \\
13 & Pulsatrix perspicillata & LC & II & \\
\hline
\end{tabular}

\section{Conclusiones}

$\mathrm{S}_{\mathrm{e}}^{\mathrm{e}}$ registraron 94 especies de aves en la zona de estudio como resultado preliminar de una alta diversidad en el CIPCA, debido a las condiciones ambientales y ecológicas de sus ecosistemas.

La composición de aves en los dos estratos analizados fue diferente con respecto al número de especies, siendo la más abundante la zona intervenida, posiblemente debido a la presencia de árboles frutales e insectos de los agroecosistemas asociados mediante forrajeo en los bordes, comportamiento común en diversas especies de aves.

Las curvas de acumulación de especies indicaron un alto esfuerzo de monitoreo durante el estudio, constituyendo una representatividad considerable en la abundancia de aves; pudiendo registrar a largo plazo un mayor número de especies en el Cipca.

El bosque secundario demostró ser el más diverso de acuerdo a la equitatividad de las especies, tal vez debido a la menor intervención antrópica y a la regeneración de nuevos hábitats.

Se encontraron 13 especies en categorías de riesgo en los dos estratos de bosque analizados, de las cuales Tinamus tao, Ramphastos vitellinus, Ramphastos tucanus y Lepidothrix coronata se encuentran en un estado más vulnerable, y deben ser consideradas para implementación de estrategias de conservación.

\section{Bibliografía}

Borojes, J. y Lopez, L. (2005). Riqueza y diversidad de aves en una selva mediana subperennifolia en el centro de Veracruz, Mexico. Acta Zoologica Mexicana, 21(1), $1-20$.

CITES (2018). Convencion sobre el Comercio Internacional de Especies Amenazadas de Fauna y Flora Silvestres. Obtenido de https://cites.org/esp/app/index.php

CMS (2018). Convencion sobre las especies migratorias de animales silvestres. Recuperado el 15 de 02 de 2018, de https://www.cms.int/es/page/texto-dela-convenci\%C3\%B3n

Colwell, R. (2013). Statistical estimation of species richness and shared species from samples. [En línea].

Daily, G. C. (1997). Nature's Services: Societal Dependence on Natural Ecosystems. Washington, DC: Island Press, $392 \mathrm{p}$.

Enriquez, L. y Saenz, M. L. (2006). Riqueza y adundancia de aves y su relación con la cobertura arbórea en un agropaisaje dominada por la ganadería en el trópico de subhumedo de Costa Rica. Agroforesteria en las Americas, 45, 49-57

Freile, J. (2019). Aves del Ecuador Version 2019. Obtenido de Museo de Zoología, Pontificia Universidad Católica del Ecuador: https://bioweb.bio/faunaweb/avesweb

Freile, J., Brinkhuizen, D., Greenfield, P., Navarrete, L. y Nilsson, J. (2017). Checklist of the Birds of Ecuador:Comite Ecuatoriano de Registros Ornitologicos. Quito: http//ceroecuador.wordpress.com. Losada-Prado, S., González-Prieto, A., Carvajal, A. y , Y. G. Molina-Martínez. (2005). Especies endémicas y amenazadas, registradas en la cuenca del Río Coello (Tolima) durante estudios rápidos. Revista Ornitología Colombiana 3, 76-80. 
Ruiz, J. R. L. (2015). Determinación de la potencialidad turística de la avifauna de la comunidad 23 de Noviembre, Naranjal, Ecuador. Revista Interamericana de Ambiente y Turismo-RIAT, 11(2), 163-173.

MAE (2012). Ministerio del Ambiente del Ecuador. Sistema de clasificación de los ecosistemas del Ecuador continental. Subsecretaría de Patrimonio Natural. Quito.

McMullan, M., y Navarrete, L. (2010). Fieldbook of the Birds of Ecuador incluiding the Galapagos Islands. Quito: Colibri Digital.

Mindo Cloud Fundation (20 de Febrero de 2017). Actualizacion de la Estrategia Nacional de Aviturismo. Obtenido de Ministerio del Turismo del Ecuador: https:// mindocloudforest.org/

Molina-Martinez, Y., García-Melo, J. y Losada-Prado, S. (2015). Evaluación rápida de las aves de la parte baja de la cuenca del río Anamichú, municipio de Rio Blanco -Tolina. Revista Tumbaga, 2 (10), 72-93.

Moore, J. V., Krabbe, N. y Jahn O. (2003). Bird sounds of Ecuador. San Jose, California: John V. Moore Nature Recordings.

Naranjo, L. (1998). Diversidad Ecosistémica. Instituto de Recursos Biológicos Alexander von Humbolt, 535 pp.

Niemi, G., y McDonald, M. (2004). Aplicaton of ecological indicators. Annual of ecology, evolution an systematics, 35 (1), 89-111.

Øyvind , H., Harper, D. A., y Ryan, P. D. (2001). Past:Paleontological Statistics Software Package for Education and data Analysis. Paleontologia Electronica, 4(1), 4-9.

Ramirez, J. (2009). Diversidad de aves de habitats naturales y modificados en un paisaje de la depresión central de Chiapas, México. Revista universitaria de la Universidad Autonoma de México, 58(1), 1-18.

RAMSAR. (2005). Directrices para la evaluación rápida de la biodiversidad de los humedales continentales, costeros y marinos. $9^{a}$ Reunión de la Conferencia de las Partes Contratantes en la Convención sobre los Humedales (Ramsar, Irán, 1971), Resolución IX.1 Anexo E i, Kampala, Uganda.

Remsen, J., Areta, J., Cadena, C., Jaramillo, A., Nores, M. y Zimmer, K. (2017). South American Classification Committe, American Ornitologists Unión. Estados Unidos: American Ornitologists.

Ridgely, R. S. y Greenfield, P. J. (2010). Aves del Ecuador, Guía de campo. Vol 1. Colibrí Digital. Quito, Ecuador.

Sayre, R., E. Roca, G. Sedaghatkish, B. Young, S. Keel, R y Roca, S. Sheppard. (2002). Un enfoque en la naturaleza: Evaluaciones ecológicas rápidas. The Nature Conservancy, Publicaciones para la capacitación. Arlington, USA.

Stotz, D., Fitzapatrick, T., y Monoskivits D. K. (1996). Neotropical Birds: Ecology and Conservation. The university of Chicago Press, 78(5).
UEA (2018). Universidad Estatal Amazonica. Recuperado el 18 de marzo del 2018, https://www.uea.edu.ec/cipca/

UICN (2016). Guidelines for using the UICN Red List Categories and criteria. Standars and petitions, Subcomite of the IUCN y SSC Red List Programme Committe. Gland.UK:UICN

Villareal, H., Alvarez, M., Córdoba, S., Escobar, F., Fagua, G., Gast., F., et al. (2004). Metodos para el analisis de datos: una aplicacion de resultados proveniente de caracterizaciones de biodiversidad. En: Manual para el desarollo de inventarios de biodiversidad. Instituto de Investigacion de Recursos Biológicos Alexander von Humbolt. Bogotá, Colombia. 236 pp. 


\title{
FNCS: Propuesta de una plataforma de gestión de dispositivos de Red basados en RouterOS
}

\author{
NCS: Proposal of a platform for the management of Network devices based on RouterOS
}

\author{
Alberto Nuñez Agurto \\ ${ }^{1}$ Departamento de Ciencias de la Computación, Universidad de las Fuerzas Armadas ESPE - Sede Santo Domingo, \\ adnunez1@espe.edu.ec
}

Rec.: 19.03.2019. Acept.: 21.01.2020. Publicado el 30 de junio de 2020

\section{Resumen}

$\mathrm{E}$ n las organizaciones, contar con la disponibilidad de los servicios de red inalámbrica es imprescindible. Sin embargo, esta disponibilidad se ve afectada debido principalmente a dos factores: la necesidad de un experto en la configuración de los equipos y al desplazamiento de dicho experto al sitio, para que este configure cada uno de los equipos que necesitan dicha configuración. Por otra parte, la empresa Mirotik dispone de un Sistema Operativo (SO) para equipos Routerboard, denominado RouterOS (RouterBoard Operating System). Este SO soporta la comunicación mediante los protocolos telnet, ssh, pero las aplicaciones que utilizan estos protocolos por lo general no tienen una interfaz gráfica, además existen herramientas propietarias gráficas como winbox y webfig para configurar estos equipos, pero esto trae consigo otro tipo de problemas como son: configuración manual de equipo en equipo, mayor uso del CPU de los dispositivos, y consumo de más ancho de banda para poder gestionarlos. Para solucionar estos problemas, ha sido desarrollado una aplicación con interfaz web, denominada Fast Network Config System (FNCS), la cual permite gestionar y configurar de forma gráfica, remota y centralizada los dispositivos con RouterOS, de manera mucho más eficiente que las soluciones disponibles. Para desarrollar la aplicación, se aprovechó la API-Mikrotik que está integrada en RouterOS. Posteriormente, se comparó la velocidad en la configuración de la herramienta propuesta FNCS versus Winbox y ssh, y se comprobó que la solución FNCS es hasta 200 veces más rápida.

Palabras clave: Mikrotik, RouterOS, API-Mikrotik, virtualización.

\begin{abstract}
Tn organizations, having the availability of network services is essential. However, this availability is affected mainly due to two factors: the need for an expert in the configuration of the equipment and the movement of this expert to the site, so that it configures each of the devices that need such configuration. On the other hand, Mirotik has an Operating System (OS) for Routerboard equipment, called RouterOS (RouterBoard Operating System). This OS supports communication through the protocols telnet, ssh, but the applications that use these protocols generally do not have a graphical interface, there are also proprietary graphic tools such as winbox and webfig to configure these devices, but this brings with it other problems such as: manual configuration of device in device, greater use of the CPU of the devices, and consumption of more bandwidth to manage them. To solve these problems, has been developed an application with web interface, called Fast Network Config System (FNCS), which allows to manage and configure graphically, remotely and centrally the devices with RouterOS, much more efficiently than the solutions available. To develop the application, we took advantage of the API-Mikrotik that is integrated in RouterOS. Subsequently, the speed was compared in the configuration of the proposed tool FNCS versus winbox and ssh, and it was found that the solution FNCS is up to 200 times faster.
\end{abstract}

keywords: Mikrotik, RouterOS, API-Mikrotik, virtualization. 


\section{Introducción}

$\mathrm{E}$ n las organizaciones, contar con la disponibilidad de los servicios de red es de suma importancia, esto implica que las organizaciones destinen gran parte del personal de tecnologías de la información (TI) a resolver problemas de infraestructura, sobre todo a nivel de acceso. El personal que se dedica a este tipo de soporte, debe tener un alto grado de habilidades en la gestión de dispositivos de red. En la mayoría de los casos, las configuraciones se vuelven complejas, debido a la cantidad de instrucciones que se ejecutan en los dispositivos, provocando que en ocasiones se cometan errores en las configuraciones y, por lo tanto, el detectarlos y corregirlos puede llevar más tiempo que la misma configuración.

La empresa Mikrotik, ha desarrollado una placa base llamada Routerboard, esta placa base permite desarrollar diferentes equipos routers, access point (AP), switchs con la integración interfaces ethernet $\mathrm{y}$ wireless, ademas integra RouterOS (Mikrotik, 2015). Existen herramientas desarrolladas por la empresa mikrotik y herramientas tradicionales, que permiten la configuración de equipos con RouterOS. Entre las herramientas propietarias tenemos winbox, que tiene una interfaz sencilla para realizar la administración y webfig, una herramienta basada en la web, por lo tanto, no necesita ninguna instalación (Mikrotik, 2018a). Existen otras herramientas tradicionales que RouterOS admite en su configuración como telnet y ssh, pero que no tienen una interfaz gráfica para realizar la gestión de su SO (Galbraith, Dyke, \& Bright, 2007). Si bien las herramientas propietarias permiten la configuración visual de los dispositivos, esto trae consigo otro tipo de problemas como son: configuración manual de equipo en equipo, mayor uso del CPU de los dispositivos, y más capacidad de ancho de banda para gestionarlos remotamente. Sin embargo, RouterOS ofrece una alternativa que permite desarrollar aplicaciones a medida del usuario para su administración y control; la cual se conoce como API-Mikrotik (Mikrotik, 2018b). Esta alternativa, permite que las organizaciones integren aplicaciones a medida, como un módulo adicional en sus aplicaciones en la mesa de soporte.

El principal objetivo del presente trabajo es agilizar la gestión de dispositivos RouterOS, mediante el diseño e implementación de una aplicación, que permita la gestión centralizada de dispositivos, aprovechando la capacidad de su comunicación a través de la APIMikrotik (Application Programmable Interface). La aplicación desarrollada se denominada FNCS (Network Config System), y permite realizar la configuración y auditoria de dispositivos basados en RouterOS de forma gráfica, remota y centralizada. El resto de este documento se ha organizado de la siguiente manera: En la Sección 2, se explica la metodología, en la Sección 3 se realiza una descripción de la aplicación y su funcionamiento después de haberla probado en un entorno virtual, en la Sección 4, se muestran los resultados de rendimiento del sistema analizados por los autores y finalmente, en la Sección 5 se exponen las conclusiones del trabajo, así como trabajos futuros.

En cuanto a trabajos relacionados con la gestión de dispositivos con RouterOS, podemos mencionar que He \& Cao (2010) proponen la implementación de un servidor PPPoE basado en RouterOS, abandonando las vias convencionales para construir de alto rendimiento, así como la dependencia del router con hardware de gama alta, pudiendo ser implementadas en grandes y medianas empresas. En el trabajo de los autores (Cheng, Wu, Routeros, Security, \& Virus, 2010), se hacen pruebas implementando PPPoE (Point-to-Point Protocol over Ethernet) sobre RouterOS, con el objetivo de controlar los problemas de seguridad inmersos en las gestión de la red, la implementación la realizan el campus del Instituto de Huangshi.

Cueva, Pozo, \& Iturralde (2017) desarrollan e implementan un programa multiplataforma, llamado ENDS (Easy Network Designer Software), que permite realizar la virtualización de la red y la configuración remota de los parámetros básicos del equipo MikroTik con RouterOS, a través de una interfaz gráfica. El software fue escrito en Java y utiliza una base de datos MySQL donde se guardan los proyectos.

Por su parte, Dolnák \& Litvik (2016), realizan pruebas complejas de conmutación por error y balanceo de carga en routers MikroTik con RouterOS, basados en estándares y utilizando la tecnología OpenVPN. Iswadi, Adriman, \& Munadi (2019) se centran en los algoritmos de gestión de ancho de banda PCQ - HTB de conmutación adaptativa con RouterOS y analizan las debilidades y ventajas de estos dos algoritmos, para proponer mecanismos de conmutación adaptativos, con el propósito de organizar el uso del ancho de banda.

\section{Materiales y métodos}

\section{Sistema operativo RouterOS}

Una de las características importantes de RouterOS, es que permite virtualizar una $\mathrm{PC}$ como si fuese un router, es decir con todas sus características; firewall, access point, bandwidth managment, hotspot Gateway y servidor VPN. Además, dispone de algunos puertos $\mathrm{y}$ protocolos, que son utilizados por determinados servicios. En el Cuadro 1se observa el listado de los servicios con sus respectivos puertos y protocolos 